\title{
GPU-acceleration of A High Order Finite Difference Code Using Curvilinear Coordinates
}

\author{
Marco Kupiainen \\ Swedish Meteorological and \\ Hydrological Institute \\ Norrköping, Sweden \\ marco.kupiainen@smhi.se
}

\author{
Jing Gong ${ }^{\dagger}$ \\ KTH Royal Institute of \\ Technology \\ Stockholm, Sweden \\ gongjing@kth.se
}

\author{
Lilit Axner \\ KTH Royal Institute of \\ Technology \\ Stockholm, Sweden \\ lilit@kth.se
}

\author{
Erwin Laure \\ KTH Royal Institute of Technology \\ Stockholm, Sweden \\ erwinl@kth.se
}

\author{
Jan Nordström \\ Linköping University \\ Linköping, Sweden \\ jan.nordström@liu.se
}

\begin{abstract}
GPU-accelerated computing is becoming a popular technology due to the emergence of techniques such as OpenACC, which makes it easy to port codes in their original form to GPU systems using compiler directives, and thereby speeding up computation times relatively simply. In this study we have developed an OpenACC implementation of the high order finite difference CFD solver ESSENSE for simulating compressible flows. The solver is based on summation-by-part form difference operators, and the boundary and interface conditions are weakly implemented using simultaneous approximation terms. This case study focuses on porting code to GPUs for the most time-consuming parts namely sparse matrix vector multiplications and the evaluations of fluxes. The resulting OpenACC implementation is used to simulate the Taylor-Green vortex which produces a maximum speed-up of 61.3 on a single V100 GPU by compared to serial CPU version.
\end{abstract}

\section{CCS CONCEPTS}

- Computing methodologies $\rightarrow$ Parallel programming languages; - Applied computing $\rightarrow$ Engineering;

- Mathematics of computing $\rightarrow$ Mathematical software performance

\section{KEYWORDS}

OpenACC, GPU programming, Computational fluid dynamics, High order finite difference method

ACM Reference format:

†Corresponding Author: gongjing@kth.se

(c) 2020 Association for Computing Machinery. ACM acknowledges that this contribution was authored or co-authored by an employee, contractor or affiliate of a national government. As such, the Government retains a nonexclusive, royaltyfree right to publish or reproduce this article, or to allow others to do so, for Government purposes only.

CNIOT2020, April 24-26, 2020, Sanya, China

(C) 2020 Association for Computing Machinery.

ACM ISBN 978-1-4503-7771-3/20/04 ..\$15.00

https://doi.org/10.1145/3398329.3398336
Marco Kupiainen, Jing Gong, Lilit Axner, Erwin Laure, and Jan Nordström. 2020. GPU-acceleration of a High Order Finite Difference Code using Curvilinear Coordinates. In Proceedings of 2020 International Conference on Computing, Networks and Internet of Things (CNIOT'20). Sanya, China, 7 pages. https://doi.org/10.1145/3398329.3398336

\section{Introduction}

ESSENSE [1, 2] is a computational fluid dynamics (CFD) solver for compressible Navier-Stokes equations using a high order finite difference method (HOFDM). The solver can handle multiple structured blocks with coupling terms between the blocks. All the techniques employed in the code are based on the summation-bypart (SBP) form of the operators and we impose the boundary and interface conditions weakly using Simultaneous Approximation Terms (SAT). With the implementation of the SAT technique, weak boundary conditions are applied on all boundaries including walls, far-field boundaries, and interface conditions between blocks [3, 4]. A common feature for all boundary and interface conditions is that they are enforced through penalty terms proportional to the difference between the unknown quantity and the corresponding prescribed data. In the solver, the governing equations are time integrated explicitly using the additive fourth order Runge-Kutta scheme.

Heterogeneous HPC architectures are increasingly prevalent in the Top500 lists [5]. There were 145 systems with nodes based on CPUs (Central Processing Units) and enhanced by accelerators or co-processors optimized by floating-point calculation in the latest version of the list, which is an increase from 134 such systems six months ago. When using NVIDIA GPU (Graphics Processing Unit) accelerators, applications are typically rewritten in a lowlevel language such as CUDA or OpenCL. In contrast, the use of OpenACC [6] enables existing HPC application codes to run on accelerators with minimal source code changes. This is achieved using compiler directives and API calls for generating optimized codes. It is up to the user, guided by performance to insert these directives where necessary. 
Some CFD codes have been taking advantage of the strong performances of GPUs. In [7] the CFD code OVERFLOW for compressible flows was accelerated on a single GPU using CUDA. A speed-up of 2 .5-3 was reported in [7] for the Symmetric successive over-relaxation (SSOR) solver in the code can be achieved. The SIMPLE (semi-implicit method for pressure linked equations) and PISO (pressure implicit with operator splitting) algorithms for CFD simulations were ported in [8] to a GPU architecture using CUDA. The results showed that the speed of the GPU version of the SIMPLE and PISO algorithms can be increased by up to a factor of 4 in comparison with the standard algorithms used in OpenFOAM. CFD solver for the compressible Navier-Stokes equations based on a third-order reconstructed discontinuous Galerkin method, which was parallelized on a single GPU using OpenACC directives was presented in [9]. OpenACC parallelization techniques, applied to an aeroacoustics simulation code, are addressed in [10] for $\mathrm{C}++$ codes. [11] accelerated a 2D solver for the Euler equations based on the MUSCL and NND algorithms on GPUs. That paper also discussed some optimization strategies for CPU/GPU heterogeneous systems. In $[12,13]$ we demonstrated that OpenACC works well for Nek5000, a high order spectral element CFD solver for incompressible flows.

\section{Theoretical Background}

The non-linear compressible Navier-Stokes equations in three dimensions in conservation form with suitable initial and boundary conditions are written in non-dimensional form (1)

$$
\begin{aligned}
\partial_{t} \boldsymbol{u} & +\partial_{x} \underbrace{\left[\boldsymbol{F}_{I}(\boldsymbol{u})-\frac{1}{R e_{a}} \boldsymbol{F}_{v}\left(\boldsymbol{u}, \partial_{x} \boldsymbol{u}, \partial_{y} \boldsymbol{u}, \partial_{z} \boldsymbol{u}\right)\right]}_{\boldsymbol{F}} \\
& +\partial_{y} \underbrace{\left[\boldsymbol{G}_{I}(\boldsymbol{u})-\frac{1}{R e_{a}} \boldsymbol{G}_{\boldsymbol{v}}\left(\boldsymbol{u}, \partial_{x} \boldsymbol{u}, \partial_{y} \boldsymbol{u}, \partial_{z} \boldsymbol{u}\right)\right]}_{\boldsymbol{G}} \\
& +\partial_{z} \underbrace{\left[\boldsymbol{H}_{I}(\boldsymbol{u})-\frac{1}{R e_{a}} \boldsymbol{H}_{v}\left(\boldsymbol{u}, \partial_{x} \boldsymbol{u}, \partial_{y} \boldsymbol{u}, \partial_{z} \boldsymbol{u}\right)\right]}_{\boldsymbol{H}} \\
& =\boldsymbol{Q}(\boldsymbol{u}, t, x, y, z)
\end{aligned}
$$

where $\boldsymbol{F}_{I}, \boldsymbol{G}_{I}$, and $\boldsymbol{H}_{I}$ are the inviscid fluxes; $\boldsymbol{F}_{v}, \boldsymbol{G}_{v}$, and $\boldsymbol{H}_{v}$ are the viscous fluxes; $\boldsymbol{Q}$ is a forcing term. The computational domain $\Omega$ dictates what boundary conditions are used along the boundary $\partial \Omega$. By denoting the coordinate transformation

$$
\xi=\xi(x, y, z), \eta=\eta(x, y, z), \zeta=\zeta(x, y, z), \xi, \eta, \zeta \in[0,1]
$$

the Navier-Stokes equations (1) can be transformed from Cartesian coordinates to general curvilinear coordinates as

$$
\partial_{t} \boldsymbol{u}+\partial_{\xi} \widetilde{\boldsymbol{F}}+\partial_{\eta} \widetilde{\boldsymbol{G}}+\partial_{\zeta} \widetilde{\boldsymbol{H}}=\widetilde{\boldsymbol{Q}}
$$

These equations are discretized onto a structured grid. In ESSENSE the SBP operator augmented with weak imposition of boundary and interface conditions by the SAT, is used $[14,15]$. This means that Equation ( 1$)$ in $\xi, \eta$, and $\zeta$ coordinates is:

$$
\begin{aligned}
\partial_{t} \boldsymbol{u} & +\partial_{x}\left(\xi_{x} \boldsymbol{F}+\xi_{y} \boldsymbol{G}+\xi_{z} \boldsymbol{H}\right) \\
& +\partial_{y}\left(\eta_{x} \boldsymbol{F}+\eta_{y} \boldsymbol{G}+\eta_{z} \boldsymbol{H}\right) \\
& +\partial_{x}\left(\zeta_{x} \boldsymbol{F}+\zeta_{x} \boldsymbol{G}+\zeta_{x} \boldsymbol{H}\right) \\
= & \boldsymbol{Q}(\boldsymbol{u}, t, x(\xi, \eta, \zeta), y(\xi, \eta, \zeta), z(\xi, \eta, \zeta)) \\
& +\sum_{i=1}^{\text {nbc+ninter }} \sigma_{i} \boldsymbol{S A \boldsymbol { T }}{ }_{i}
\end{aligned}
$$

where the last term in Equation (4) collects all the weak implementations on the boundaries and interfaces between blocks.

The differential operators are approximated in Equation (3) using the high-order SBP operators, i.e.,

$$
\partial_{\xi}=\left(\boldsymbol{P}_{\xi}^{-1} \boldsymbol{Q}_{\xi} \otimes \boldsymbol{I}_{\eta} \otimes \boldsymbol{I}_{\zeta}\right)+\mathcal{O}\left(\Delta \xi^{p}\right)
$$

where $\boldsymbol{P}_{\xi}$ is a diagonal positive definite matrix and $\boldsymbol{Q}_{\xi}+\boldsymbol{Q}_{\xi}^{\boldsymbol{T}}=$ $\operatorname{diag}(-1,0, \ldots, 0,1)[14]$. Notice that all metric derivatives multi- plying the factor $1 / \operatorname{det}(\boldsymbol{J})$, e.g.

$$
\xi_{x}=\frac{1}{\operatorname{det}(\boldsymbol{J})}\left(y_{\eta} z_{\zeta}-y_{\zeta} z_{\eta}\right)
$$

with the Jacobian matrix $\boldsymbol{J}$ defined as

$$
\boldsymbol{J}=\left[\begin{array}{lll}
x_{\xi} & x_{\eta} & x_{\zeta} \\
y_{\xi} & y_{\eta} & y_{\zeta} \\
z_{\xi} & z_{\eta} & z_{\zeta}
\end{array}\right]
$$

with the computation in a way that respect the geometric conservation law, see [16]. Consequently, in curvilinear coordinates, Equation (4) involves in heavy intensive computations of sparse matrix vector multiplications when the metric derivatives namely $\partial / \partial \xi, \partial / \partial \eta$, and $\partial / \partial \zeta$ are being calculated.

The pseudo-code with the explicit Runge-Kutta method of ESSENSE is presented in Algorithm 1. For each step of the main loop, the explicit fourth order Rung-Kutta method with 4 stages has been implemented. The sub-stages of the Runge-Kutta method consist of the main computation kernels, i.e., calculating the inviscid and viscous fluxes, applying the artificial dissipation, applying the body forces and finally updating the variables for density, velocities, and pressure. It is important to note that the metric derivatives $\partial / \partial \xi, \partial / \partial \eta$, and $\partial / \partial \zeta$ in the corresponding subroutines diff_xi, diff_eta, and diff_zeta in the code, respectively, are highly involved in all these computation kernels. This can be seen from Equation (4).

\section{OpenACC Implementation and Optimization}

\subsection{Profiling Analysis}

An initial performance profiling of the ESSENSE solver on a single CPU has been carried out using the PGI profiler pgprof. The goal of this profiling work was to identify which subroutines are the most time consuming, and provide enough workload to exploit the GPU computational power. We used a sampling experiment which samples the program counter at a given 
frequency. The sampling experiment reports an exclusive statistic for each function and its accuracy depends on the sampling frequency. Table 1 shows the profiling results of the most timeconsuming subroutines for a 3D case with $65 \times 65 \times 65$ grid points on a single Intel Broadwell CPU core.

Algorithm 1 The pseudo-code of ESSENSE.

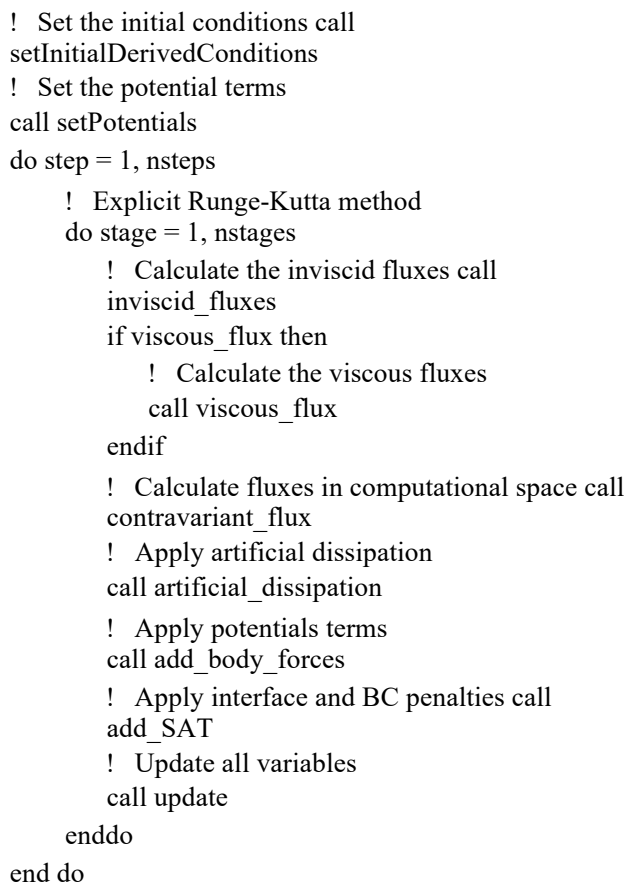

Table 1: Profiling results of ESSENSE on a single Intel Broadwell CPU core with the PGI profiler pgprof for a case of $65^{3}$ grid points.

\begin{tabular}{lc}
\hline Subroutine & Exclusive Execution Time (\%) \\
\hline diff_xi & $21.91 \%$ \\
diff_eta & $19.64 \%$ \\
diff_zeta & $9.45 \%$ \\
gradient_cons & $10.92 \%$ \\
add_flux_contribution & $4.15 \%$ \\
artificial_dissipation & $2.65 \%$ \\
viscous_flux & $1.43 \%$ \\
\hline
\end{tabular}

Naturally the subroutines with the higher exclusive counts execute for longer times, which causes performance bottlenecks. It is clear from the profiling results shown in Table 1 that the subroutines diff_xi, diff_eta, and diff_zeta for calculating the sparse matrix vector multiplications require approximately $51.0 \%$ of the total execution time. The exclusive execution time for the other subroutines (gradient_cons, add_flux_contribution, viscous_flux, and artificialdissipation) for evaluating the convective and viscous fluxes require approximately $19.1 \%$ of the total execution. The sparse matrix vector multiplications and the evaluation of fluxes dominate the execution time of ESSENSE (requiring around $70.1 \%$ of the time).

\subsection{The Sparse Matrix Vector Multiplications}

As outlined in Section 2, the metric derivatives $\partial / \partial \xi, \partial / \eta$, and $\partial / \partial \zeta$ for the three-dimensional curvilinear coordinates has been implemented in the code as sparse matrix vector multiplications (SpMV). The SpMV has been formalized as $y=A x$, where $A$ is a sparse matrix, $x$ and $y$ are two dense vectors. The sparse matrix can be stored in many formats, such as compressed sparse row (CSR), com-pressed sparse column (CSC), coordinate (COO), diagonal (DIA), or Ellpack-Itpack(ELL). The CSR format has been chosen in ESSENSE. The CSR format requires three arrays: one array contains non-zero values in the form of real numbers; one array consists of integers storing the corresponding column indexes, and the other array contains integers representing the offsets for where each row starts.

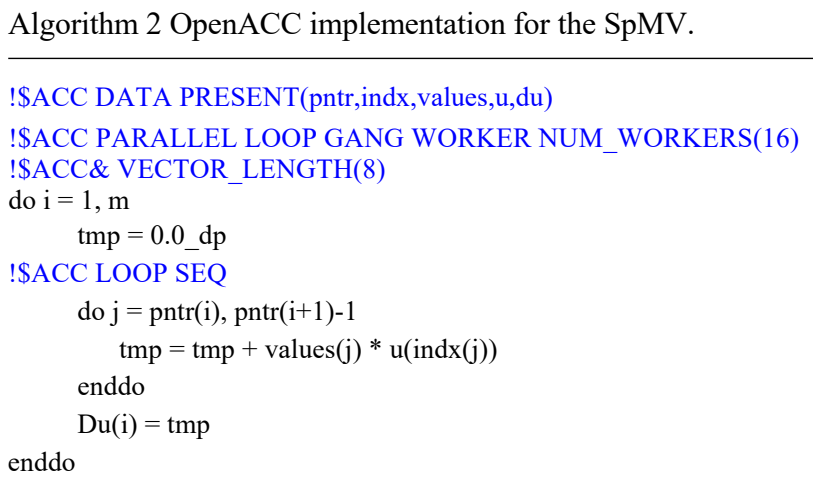

One version of the SpMV with OpenACC directives is shown in Algorithm 2. The SpMV has been characterized by low operational intensity and many factors significantly affect the performance of SpMV evaluations [17]. With the PGI compiler, we used the OpenACC clauses NUM_WORKERS and VECTOR_LENGTH to manually control the occupancy in order to improve the performance of the SpMV. It has been reported that VECTOR_LENGTH is mainly dependent on the number of nonzeros values in a typical row of the matrix [6]. To obtain good performance on GPUs, the VECTOR_LENGTH of 8 is chosen to match the 8 entries per row for the stencil of the 8-th order SBP operator. The OpenACC directive \$ACC LOOP SEQ has been used in the inner-loop to avoid parallelization within threads. 
Because we observed that the OpenACC clause REDUCTION(+:sum) and ATOMIC directive performed worse for our cases in practice, they were not used in the studies.

One of the features of the OpenACC Application Program Interface (API) is that the CUDA native device libraries are callable using the OpenACC programming models. An example of API for the library cuSPARSE is presented in Algorithm 3. The OpenACC directive HOST_DATA and USE_DEVICE clause are used to pass the references to accelerator memory to the library instead of the host memory [11]. The CUDA cuSPAPSE library consist of a set of linear algebra subroutines of handling the operators of sparse matrices. In Algorithm 3 the subroutine cusparseDcsrmv is called to execute the SpMV operator where the matrix is defined in CSR storage format.

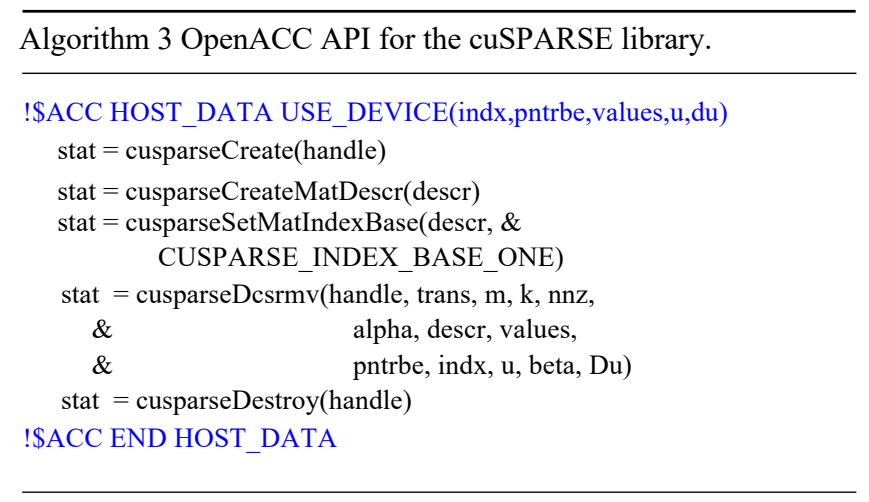

\subsection{Parallelization of Other Kernels}

The evaluation of convective and viscous fluxes is the other part of the code that requires a large execution time, see Table 1, where large-scale matrices are involved. A typical operator with large matrices involved in three dimensions is shown in Algorithm 4, where $n_{i}, n_{j}$, and $n_{k}$ are the number of grid points in $x$-, $y$-, and $z$-dimensions with typical sizes from $32-256$. The process of porting to OpenACC is achieved by converting one routine at a time. Since the structure of the matrix process

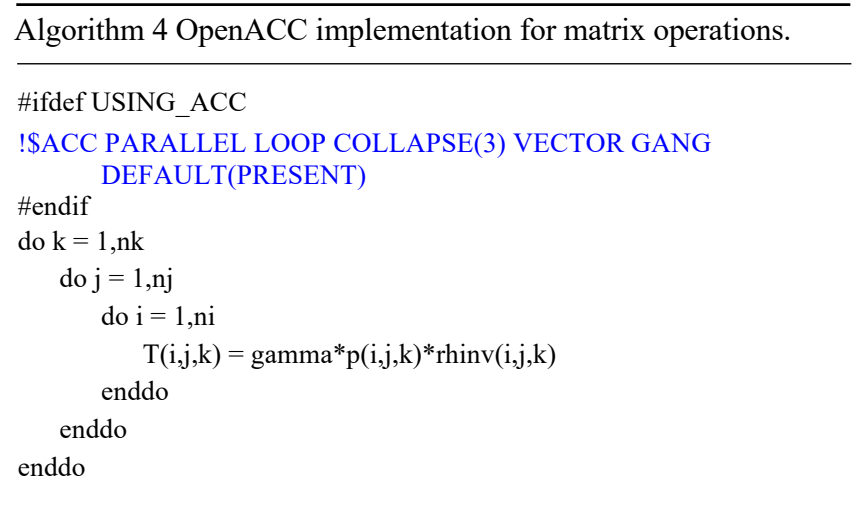

consists of multidimensional loops that are tightly nested with each loop being completely independent of every other one, the OpenACC clause COLLAPSE(3) is used to enable the compiler to turn the next three loops into one flattened loop. In such cases, this clause can help improve performance by exposing more parallelism: DEFAULT(PRESENT) is used to explicitly instruct that all data should be present on the devices without any data between the host and devices, see Algorithm 4. As a result, the program will crash with run-time errors if any data is still on the host.

In order to avoid a significant modification of the original code, we have added ACC ROUTINE directive to instruct the compiler to build a device version of subroutine, which can be called from an OpenACC parallel loop, see Algorithm 5.

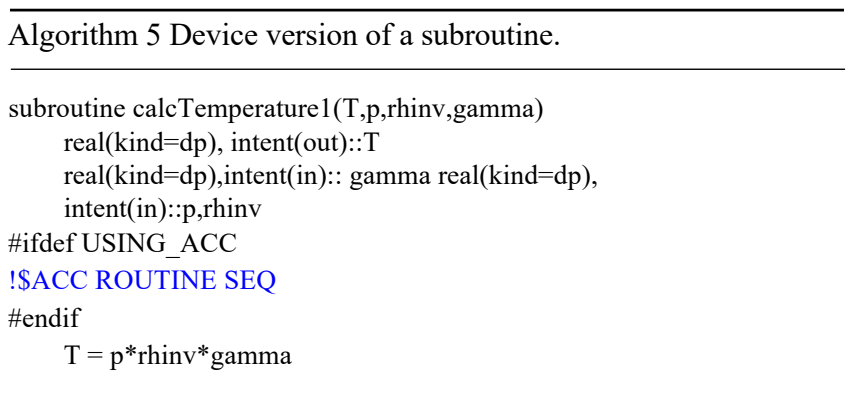

\subsection{Data Management}

To reduce data movement between hosts and devices, we have created data regions using the OpenACC data directive, by combining OpenACC unstructured enter data and exit data directives, as well as the PRESENT data clause. Then we have indicated to the compiler that the data is already present in the device memory in order to keep all the data on the device operated by GPU. This would allow for data to be initialized on the device at the start of simulation, and for data to be retained in the device memory for the lifetime of the application [18]. In this OpenACC implementation, most arrays and multi-dimensional pointers including temporary arrays have been created only once in the device global memory in the subroutines setInitialDerivedConditions in Algorithm 6 before the

Algorithm 6 The data transfer between GPU and CPU.

!\$ACC ENTER DATA COPYIN(Op\%Dop\%values,Op\%Dop\%columns)

!\$ACC\& COPYIN(Op\%Dop\%pointer)

!\$ACC DATA CREATE(F,G,H,Fv,Gv,Hv,Fxi,Geta,Hzeta)

!\$ACC\& CREATE(Ftot,Gtot,Htot,FartVisc,GartVisc,HartVisc)

!\$ACC ENTER DATA COPYIN(el\%p,el\%u,el\%rhinv)

!\$ACC ENTER DATA COPYIN(el\%gradu,el\%gradv,el\%gradw)

!\$ACC\& COPYIN(el\%gradT,physics)

!\$ACC ENTER DATA COPYIN(el\%mu,el\%mu_t)

!\$ACC ENTER DATA CREATE( $\mathrm{el} \% \mathrm{~T})$

!\$ACC ENTER DATA CREATE(Au)

main loop. After that, these arrays and pointers will be reused and updated in the device global memory. When the preset time steps 
or convergence conditions have been achieved, the variables in the device memory will be updated (copyout) to the host. In addition, ESSENSE has FORTRAN F95 features with objectoriented programming models. With the data movement of multidimension pointers within the object-oriented programming models, the compiler must assume that other pointers point to the same memory, thus making the inner loops non-parallelizable. We have used the CUDA Unified Memory (-ta=tesla:managed) for the deep copy in the port. The CUDA Unified Memory does have the caveat that only dynamic data (allocatable) is managed [18].

With the CUDA Unified Memory, one pointer still needs to be manually managed using data regions but all allocatable members of the point can be managed by the run-time, see typical data region in Algorithm 6.

\section{Performance and Numerical Results}

We evaluated the performance tests on two different GPU systems; a Cray XC40 system with NVIDIA Tesla P100 GPUs at Cray Inc. and an IBM Power9 system with NVIDIA Tesla V100 GPUs at BSC. The system configurations, with details of the hardware and software for both systems, are presented in Table 2.

Table 2: The system environment of a single node for the evaluations

\begin{tabular}{|c|c|c|}
\hline Name & Swan & CTE-POWER \\
\hline \multirow{3}{*}{$\overline{\mathrm{CPU}}$} & $\operatorname{Intel}(\mathrm{R}) \mathrm{Xeon}(\mathrm{R})$ & IBM Power9 \\
\hline & CPU E5-2697 v4@2.30GHz & 8335-GTH@2.4GHz \\
\hline & & NVIDIA Tesla \\
\hline GPU & NVIDIA Tesla P100 & $\mathrm{V} 100 \times 4$ \\
\hline CPU memory & $64 \mathrm{~GB}$ & $512 \mathrm{~GB}$ \\
\hline GPU memory & $16 \mathrm{~GB}$ & $32 \mathrm{~GB}$ \\
\hline Compiler & PGI $18.10+$ CUDA 10.0 & PGI $18.10+$ CUDA 10.0 \\
\hline
\end{tabular}

Applying the solver to the Taylor-Green vortex problem examines its ability to accurately simulate vortex decay and subsequent turbulence. The time evolution of kinetic energy and its dissipation rate were examined. The results were in good agreement with a reference solution obtained with a spectral method.

Figure 1 displays the performance results of the most timeconsuming subroutines. Figure 1(a) shows results using $65 \times$ $65 \times 65$ grid points on both systems. A speed-up of $19.8 \mathrm{x}$ can be achieved on the NVIDIA P100 while a speed-up of 26.9x can be obtained on the NVIDIA V100 GPU. Figure 1(b) shows the performance results for the same case but with a mesh twice as fine $(129 \times 129 \times 129)$. A speed-up of $28.1 \times$ for the most timeconsuming subroutines was achieved on the NVIDIA V100 GPU while a speed-up of $47.5 \mathrm{x}$ was obtained on the NVIDIA V100. In the evaluation, the output of the subroutines on the GPU is profiled by specifying the environment variable of the PGI compiler PGI_ACC_TIME to 1. Though there are some overheads associated with the profiling using the variable, the trend of GPU usages and speed-up can be reflected.

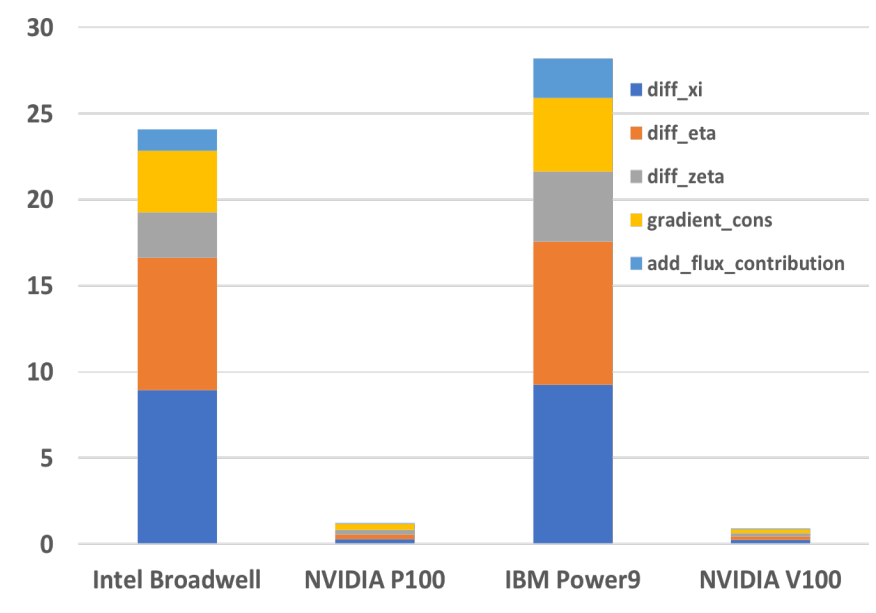

(a) $65 \times 65 \times 65$ grid points

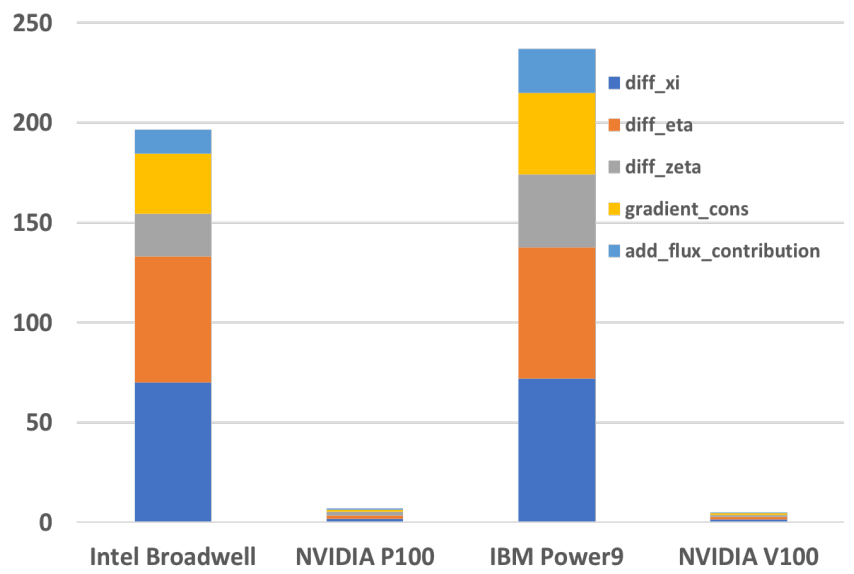

(a) $129 \times 129 \times 129$ grid points

Figure 1: The performance results of the most timeconsuming subroutines for CPU and GPU. 8-th order SBP operator used

The total execution times in seconds for 10 times-steps using different order SBP operators on different grid points are presented for the Swan system in Table 3. The speed-up with the GPU P100 increases with the number of grid points and a maximum speed-up of 38.3 can be obtained, see Figure 2. The speed-up increases from around 11 with $33^{3}$ grid points to 40 with $161^{3}$ grid points. With the $6^{\text {th }}$ order SBP, it takes $487.9 \mathrm{~s}$ on a single Intel Broadwell CPU core for 10 time-steps with $161^{3}$ grid points while it only takes 7.96 s on the P100 GPU. A similar trend for speed-up and performance can also be observed on the CTE-Power system, see Table 4 and Figure 3. For the case of 
$161^{3}$ grid points, the speed-up is $61.3 \mathrm{x}$ on NVIDIA V100 GPU in comparison to the IBM Power9 CPU core.

Table 3: The comparison of execution time in seconds for $\mathbf{1 0}$ time steps using different polynomial order on the Swan system.

\begin{tabular}{|c|c|c|c|c|c|c|}
\hline \multicolumn{3}{|c|}{$4^{\text {th }}$ order } & \multicolumn{2}{|c|}{$6^{\text {th }}$ order } & \multicolumn{2}{|c|}{$8^{\text {th }}$ order } \\
\hline \multicolumn{7}{|c|}{ Grid Points Broadwell P100 Broadwell P100 Broadwell P100 } \\
\hline $33^{3}$ & 3.8 & 0.34 & 4.4 & 0.38 & 4.7 & 0.36 \\
\hline $65^{3}$ & 27.8 & 1.24 & 32.0 & 1.33 & 33.1 & 1.34 \\
\hline $97^{3}$ & 92.6 & 2.63 & 106.3 & 2.74 & 108.8 & 3.03 \\
\hline $129^{3}$ & 217.7 & 6.16 & 249.2 & 6.65 & 255.3 & 6.92 \\
\hline $161^{3}$ & 421.9 & 11.02 & 485.8 & 12.08 & 496.4 & 12.89 \\
\hline
\end{tabular}

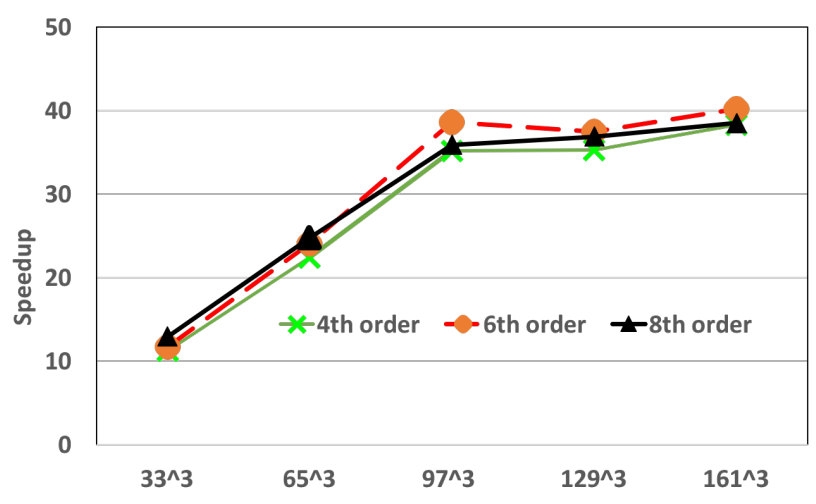

Figure 2: Speed-up on Swan system

Table 4: The comparison of execution time in seconds for $\mathbf{1 0}$ time steps using different polynomial orders on the CTEPower system.

\begin{tabular}{|c|c|c|c|c|c|c|}
\hline & \multicolumn{2}{|c|}{$4^{\text {th }}$ order } & \multicolumn{2}{|c|}{$6^{\text {th }}$ order } & \multicolumn{2}{|c|}{$8^{\text {th }}$ order } \\
\hline \multicolumn{7}{|c|}{ Grid Points Power9 V100 Power9 V100 Power9 V10 } \\
\hline $33^{3}$ & 5.4 & 0.48 & 5.9 & 0.50 & 6.5 & 0.50 \\
\hline $65^{3}$ & 33.0 & 1.06 & 36.6 & 1.16 & 39.8 & 1.20 \\
\hline $97^{3}$ & 100.9 & 2.31 & 112.9 & 2.48 & 121.8 & 2.57 \\
\hline $129^{3}$ & 231.2 & 4.45 & 259.7 & 4.89 & 276.5 & 5.10 \\
\hline $161^{3}$ & 432.2 & 7.48 & 487.9 & 7.96 & 528.3 & 8.51 \\
\hline
\end{tabular}

By using curvilinear coordinates, the higher order FDM solver can handle complex geometries. To illustrate this, Figure 4(a) presents a simulation of the temperature of mountain St. Helens, which has resolution of $100 \mathrm{~m}^{2}$ on the ground in the vertical using the ES-SENSE solver. The vortex shown in Figure 4(b) is a CFD simulation to check reproducibility of the Crow instability.

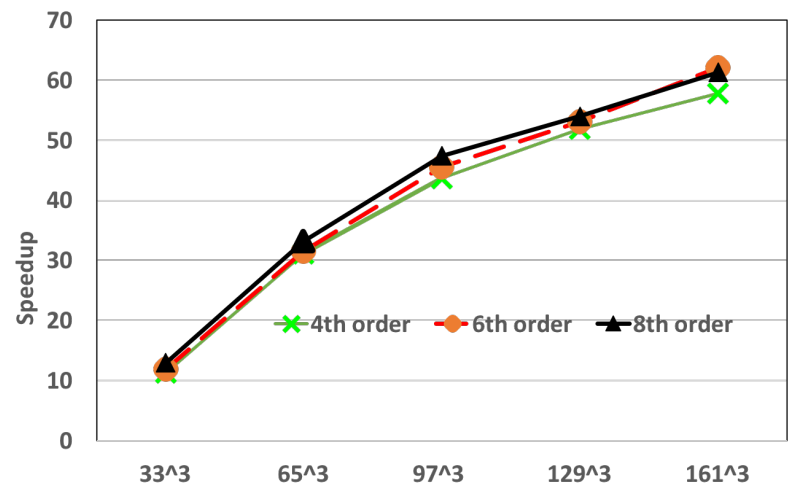

Figure 3: Speed-up on the CTE-Power system

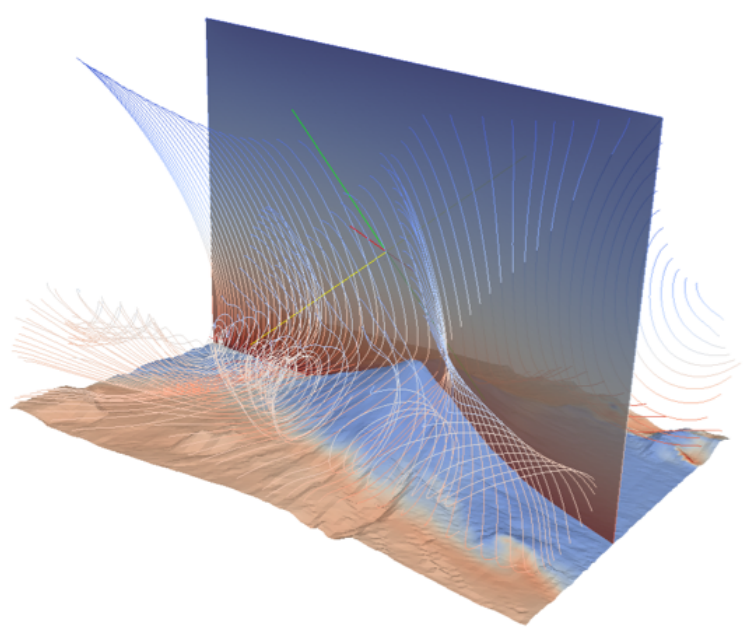

(a) Mountain St. Helens with resolution of $100^{2} \mathrm{~m}$ on the ground

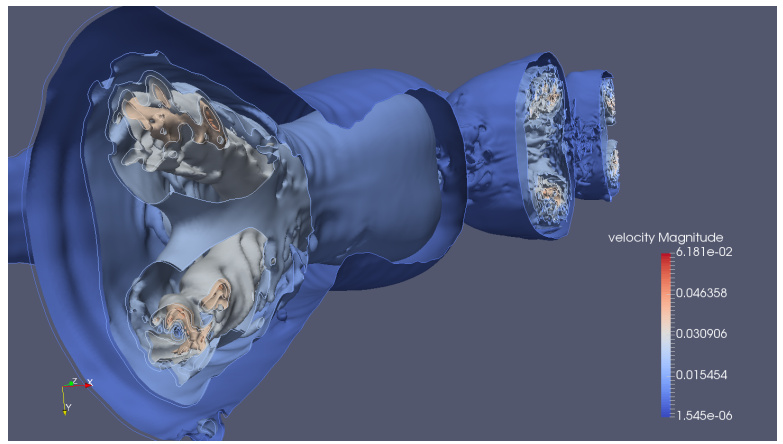

(b) Vortex simulation showing the Crow instability.

Figure 4: Simulations with curvilinear coordinates. 


\section{Conclusions and Future Works}

The ESSENSE solver has been ported to GPU systems using OpenACC compiler directives. The work was focused on porting the most time-consuming parts of ESSENSE, namely the sparse matrix vector multiplications and evaluation of inviscid and viscous fluxes, to the GPU systems. A performance analysis of the ESSENSE code using pgprof was carried out. We obtained a maximum speed-up of 61.3 using a V100 GPU in comparison to the results using the serial CPU version. In the future, we plan to implement a multi-GPU version and to continue optimizing the computation kernels.

\section{ACKNOWLEDGMENTS}

This research received funding from the Exascale Simulation Software Initiative (SESSI) in the Swedish e-Science Research Centre (SeRC) and the PRACE project which is funded in part by the EU's Horizon 2020 research and innovation programme (2014-2020) under grant agreement 653838. We are grateful for the computing time that was made available to us on the systems at Barcelona Super-computing Center (BSC) in Spain, High Performance Computing Center North (HPC2N) in Sweden, and Cray Inc.

\section{REFERENCES}

[1] Peter Eliasson, Marco Kupiainen, and Jan Nordström. 2014. Higher Order Accurate Solutions for Flow in a Cavity: Experiences and Lessons Learned, Spectral and High Order Methods for Partial Differential Equations. Lecture Notes in Computational Science and Engineering, Vol. 106. Springer-Verlag, New York, NY, 189-196.

[2] Jan Nordström and Peter Eliasson. 2015. New Developments for Increased Performance of the SBP-SAT Finite Difference Technique, Notes on Numerical Fluid Mechanics and Multidisciplinary Design. Notes on Numerical Fluid Mechanics and Multidisciplinary Design 128 (2015), 467-488

[3] David C. Del Rey Fernandez, Jason E. Hicken, and David W. Zingg. 2014 Review of summation-by-parts operators with simultaneous approximation terms for the numerical solution of partial differential equations. Computers \& Fluids 95 (2014), 171-196.

[4] Magnus Svärd and Jan Nordström". 2014. Review of summation-by-parts schemes for initial boundary value problems. J. Comput. Phy. 268 (2014), 17 38.

[5] Top500 2019. TOP500 Supercomputer Sites. Retrieved March 29, 2020 from https://www.top500.org

[6] OpenACC 2020. OpenACC 2.7 Specification. Retrieved March 29, 2020 from https://www.openacc-standard.org

[7] Dennis C. Jespersen. 2010. Acceleration of a CFD code with a GPU. Scientific Programming 18 (2010), 193-201.

[8] Tadeusz Tomczak, Katarzyna Zadarnowska, Zbigniew Koza, Maciej Matyka, and Lukasz Miroslaw. 2013. Acceleration of iterative Navier-Stokes solvers on graphics processing units. Inter. J. Comput. Fluid Dynamics 27 (2013), 201209. https://doi.org/10.1080/10618562.2013.804178

[9] Yidong Xia, Jialin Luo, Hong Luo, Jack Edwards, and Frank Mueller. 2015. OpenACC acceleration of an unstructured CFD solver based on a reconstructed discontinuous Galerkin method for compressible flows. J. Numerical Methods 78 (2015), 123-139.

[10] Marco Nicolini, Julian Miller, Sandra Wienke, Michael Schlottke-Lakemper, Matthias Meinke, and Matthias S. Müller. 2016. Software cost analysis of GPUaccelerated aeroacoustics simulations in $\mathrm{C}++$ with OpenACC. Lecture Notes in Computer Science, Vol. 9945. Springer-Verlag, New York, NY, 524-543.

[11] Jiang Lei, Da li Li, Yun long Zhou, and Wei Liu. 2019. Optimization and acceleration of flow simulations for CFD on CPU/GPU architecture. $J$. the Brazilian Society of Mechanical Sciences and Engineering 41 (2019), 123-139.

[12] Jing Gong, Stefano Markidis, Erwin Laure, Matthew Otten, Paul Fischer, and Misun Min. 2016. Nekbone performance on GPUs with OpenACC and CUDA Fortran implementations. J. Supercomputing 72 (2016), 4160-4180.
[13] Evelyn Otero, Jing Gong, Misun Min, Paul Fischer, Philipp Schlatter, and Erwin Laure. 2019. OpenACC accelerator for the Pn-Pn-2 algorithm in Nek5000. J. Parallel and Distrib. Comput. 132 (2019), 69-78.

[14] Jan Nordström, Jing Gong, Edwin van der Weide, and Magnus Svärd. 2009. A stable and conservative high order multi-block method for the compressible Navier-Stokes equations. J. Comput. Phys. 228 (2009), 9020-9035.

[15] Magnus Svärd and Jan Nordström. 2008. A stable high order finite difference scheme for the compressible Navier-Stokes equations No-slip wall boundary conditions. J. Comput. Phy. 227 (2008), 4805-4824.

[16] Marcel Vinokur. 1974. Conservation Equations of Gasdynamics in Curvilinear Coordinate Systems. J. Comput. Phy. 14 (1974), 105-125.

[17] Athena Elafrou, Georgios Goumas, and Nectarios Koziris. 2017. Performance Analysis and Optimization of Sparse Matrix-Vector Multiplication on Modern Multi- and Many-Core Processors. https://arxiv.org/pdf/1711.05487.pdf

[18] Sebastien Deldon, James Beyer, and Douglas Miles. 2018. OpenACC and CUDA Unified Memory. In Proceeding of CUG 2018. 1-9. 